\title{
PM2.5 exposure aggravates oligomeric amyloid beta-induced neuronal injury and promotes NLRP3 inflammasome activation in an in vitro model of Alzheimer's disease
}

Bian-Rong Wang ${ }^{1}$, Jian-Quan Shi ${ }^{*}$, Nian-Nian Ge², Zhou Ou², You-Yong Tian², Teng Jiang ${ }^{2}$, Jun-Shan Zhou ${ }^{2}$, Jun $\mathrm{Xu}^{3,4,5^{*}}$ and Ying-Dong Zhang ${ }^{2^{*}}$

\begin{abstract}
Background: Numerous studies suggested that PM2.5 exposure was associated with increased risk of Alzheimer's disease (AD). But the precise mechanisms by which PM2.5 contributed to AD pathogenesis have not been clarified.

Methods: In the presence or absence of neurons, oligomeric amyloid beta (OAB)-primed microglia were stimulated with PM2.5. Firstly, we determined the effects of PM2.5 exposure on neuronal injury and inflammation in neuronsmicroglia co-cultures. Then, we examined whether NLRP3 inflammasome activation was involved in PM2.5-induced inflammation. After that, we investigated whether PM2.5 exposure increased ROS level in oA $\beta$-stimulated microglia. At last, we examined whether ROS and NLRP3 inflammasome activation was required for PM2.5-induced neuronal injury in neurons-microglia co-cultures.
\end{abstract}

Results: In the present study, we showed that PM2.5 exposure aggravated oA $\beta$-induced neuronal injury and inflammation in neurons-microglia co-cultures via increasing IL-1 $\beta$ production. Further, PM2.5-induced IL-1 $\beta$ production in OA $\beta$-stimulated microglia was possibly dependent on NLRP3 inflammasome activation. Meanwhile, PM2.5 exposure increased ROS level in oA $\beta$-stimulated microglia. ROS was required for PM2.5-induced IL-1 $\beta$ production and NLRP3 inflammasome activation in oA -stimulated microglia. More importantly, ROS and NLRP3 inflammasome activation was required for PM2.5-induced neuronal injury in neurons-microglia co-cultures.

Conclusions: For the first time, these results suggested that the effects of PM2.5 under AD context were possibly mediated by NLRP3 inflammasome activation, which was triggered by ROS. Taken together, these findings have deepened our understanding on the role of PM2.5 in AD pathogenesis.

Keywords: Alzheimer's disease, PM2.5, Neuronal injury, Inflammation, NLRP3 inflammasome, ROS

\footnotetext{
*Correspondence: myxiyi_0717@163.com; neurojun1@163.com; neurojun@126.com; zhangyingdongnjmu@163.com

${ }^{2}$ Department of Neurology, Nanjing First Hospital, Nanjing Medical University, No.68, Changle Road, Nanjing 210006, Jiangsu Province, People's Republic of China

${ }^{3}$ Department of Neurology, Beijing Tiantan Hospital, Capital Medical University, No. 6 Tiantanxili, Dongcheng District, Beijing 100050, People's Republic of China

Full list of author information is available at the end of the article
}

(c) The Author(s). 2018 Open Access This article is distributed under the terms of the Creative Commons Attribution 4.0 International License (http://creativecommons.org/licenses/by/4.0/), which permits unrestricted use, distribution, and reproduction in any medium, provided you give appropriate credit to the original author(s) and the source, provide a link to the Creative Commons license, and indicate if changes were made. The Creative Commons Public Domain Dedication waiver (http://creativecommons.org/publicdomain/zero/1.0/) applies to the data made available in this article, unless otherwise stated. 


\section{Background}

Alzheimer's disease (AD) is the most common cause of dementia in the elderly. Unfortunately, the pathogenesis of AD still remains elusive [1]. There is currently an increasing interest in the association between air pollutant and AD. Air pollution is comprised of a diverse mixture of particulate matter (PM), gases, organic compounds, and metals present in outdoor and indoor air [2]. Toxic effects of environmental toxicants have been identified in in vitro and animal studies. Long-term exposures to environmental toxicants are speculated to trigger neuroinflammation and neuropathology, which paved the way for developing $\mathrm{AD}$ [3].

Of these environmental toxicants, PM poses severe health threats. PM is divided into three major size categories: ultra-fine PM (UFPM, $<0.1 \mu \mathrm{M})$, fine PM (PM2.5, $<2$. $5 \mu \mathrm{M}$ ), and coarse PM (PM10, $<10$ and $>2.5 \mu \mathrm{M}$ ). PM2.5 are mainly composed of compounds of both organic and inorganic, including sulfates, nitrates, carbon, ammonium, hydrogen ions, lipopolysaccharides (LPS), metals, and water [4]. A population-based cohort study suggested that higher concentration of PM2.5 exposure was associated with increased risk of newly diagnosed $\mathrm{AD}$ [5]. But the precise mechanisms by which PM2.5 contributed to AD pathogenesis have not been clarified.

Neuroinflammation associated with microglia has been identified as a major contributor to AD pathogenesis [6]. Long-term exposure to PM2.5 has been reported to be closely associated with neuroinflammation in human [7]. Meanwhile, several lines of evidence suggested that PM2.5 exposure aggravated neuroinflammation in the brains of mice and rats [8-11]. A pilot study suggested that prolonged exposure to PM2.5 had the potential to alter the brain inflammatory phenotype and promote the development of early AD-like pathology [12]. However, the underlying mechanisms by which PM2.5 led to neuroinflammation under AD context remained largely unclear.

The NLRP3 inflammasome is a cytoplasmic multiprotein complex that regulates the cleavage of IL-1 $\beta$ precursors. Activation of the NLRP3 inflammasome requires two signals. The first signal leads to the synthesis of pro-IL-1 $\beta$ and other components of the inflammasome, such as NLRP3. The second signal results in the assembly of the NLRP3 inflammasome, caspase- 1 activation, and IL- $1 \beta$ secretion [13]. NLRP3 inflammasome plays a pivotal role in $\mathrm{A} \beta$-induced inflammation [14]. Furthermore, NLRP3 inflammasome regulates the phenotype and function of microglia, which eventually affects amyloid beta $(A \beta)$ pathology and behavioral deficits in AD transgenic mice [15]. Hence, NLRP3 inflammasome has been regarded as therapeutic targets for $\mathrm{AD}$ [16]. On the other hand, several lines of evidence have indicated that particulate matter could induce NLRP3 inflammasome activation in airway epithelial cells $[17,18]$.

On consideration of the above evidence, we hypothesized that PM2.5 exposure aggravated oligomeric A $\beta$
(oA $\beta$ )-induced neuronal injury and inflammation in neurons-microglia co-cultures via increasing IL-1 $\beta$ production, which was mediated by NLRP3 inflammasome activation. For the first time, we show that PM2.5 exposure aggravates oA $\beta$-induced neuronal injury and inflammation in an in vitro model of AD. Meanwhile, we reveal that the aforementioned effects of PM2.5 are mediated by NLRP3 inflammasome activation. Taken together, these findings have deepened our understanding on the role of PM2.5 in AD pathogenesis.

\section{Methods \\ Reagents}

PM2.5 was purchased from the National Institute for Standards and Technology (Gaithersburg, MD, USA). A $31-42$, LPS, NADPH, lucigenin, diphenylene iodonium (DPI, NADPH oxidase inhibitor), and $N$-acetyl-L-cysteine (NAC) were purchased from Sigma-Aldrich (St. Louis, MO, USA). Z-VAD-FMK (pan-caspase inhibitor) and Z-YVAD-FMK (caspase-1 inhibitor) were purchased from Calbiochem (Gibbstown, NJ, USA). IL-1 receptor antagonist (IL-1ra) was purchased from R\&D (Minneapolis, MN, USA). MitoQ was purchased from Focus Biomolecules (Plymouth Meeting, PA, USA).

\section{Cell culture}

Pregnant C57BL/6 mice were provided by Animal Experiment Center of Nanjing First Hospital, Nanjing Medical University. All animal procedures were carried out in accordance with Chinese Association for Laboratory Animal Sciences Guide for Care and Use of Laboratory Animals and were approved by the Nanjing Medical University Experimental Animal Care and Use Committee.

Primary microglial cells were prepared from the brains of $\mathrm{C} 57 \mathrm{BL} / 6$ at postnatal day 1 as previously described [19]. Briefly, the leptomeningeal and meninges blood vessels were removed from the cortex. Cells were dissociated by trituration and cultured in DMEM containing $10 \%$ heat-inactivated fetal bovine serum, $2 \mathrm{mM} \mathrm{L-}$ glutamine and 1\% penicillin/streptomycin (Hyclone, Logan, UT, USA) for 2-3 weeks in poly-D-lysine-coated $75-\mathrm{cm}^{2}$ flasks to form a confluent glial monolayer. Half of the medium was replaced with fresh cell culture medium every 3 days. To collect microglial cells, the cultures were shaken on a rotary shaker (placed in a cell culture incubator, $37{ }^{\circ} \mathrm{C}$ and $5 \% \mathrm{CO} 2$ ) at $250 \mathrm{rpm}$ for $3 \mathrm{~h}$. The detached microglial cells were collected by centrifugation, and the enriched microglial cell suspension was plated onto poly-D-lysine-coated 6-well plates containing poly-D-lysine-coated glass coverslips. After the cells are attached, the medium was replaced with fresh culture medium. The purity of the isolated microglia was determined by immunostaining with 
antibodies against Iba1. In average, over $90 \%$ of cultured cells were immunostained with Iba1.

Primary neurons were prepared from the cortices of mouse embryos at embryonic day 17 (E17) as previously described [20]. Briefly, cortical fragments were dissociated into single cells and resuspended in neurobasal medium supplemented with $2 \%$ B27 and $1 \%$ penicillin/streptomycin. Half of the medium was replaced with fresh cell culture medium every 3 days. The purity of the culture was more than $95 \%$ as determined by NeuN-specific immunostaining (Merck Millipore, Billerica, MA, USA).

Neurons-microglia co-cultures were performed as previously described $[21,22]$. Microglia and neurons were separately seeded into 6-well transwell plates $(0.4 \mu \mathrm{m}$ pore size, Corning Costar, St Louis, MO, USA). Neurons in neuronal medium (neurobasal medium supplemented with 2\% B27 and 1\% penicillin/streptomycin) were plated in the lower chamber. Before co-culture, microglia were activated with LPS $(1 \mu \mathrm{g} / \mathrm{ml})$ for $3 \mathrm{~h}$ (LPS priming). Inserts containing microglia were washed with fresh serum-free DMEM and then plated in the upper chamber. Then, neurons-microglia co-cultures were treated with or without oA $\beta(5 \mu \mathrm{M})$. After $12 \mathrm{~h}$, neurons-microglia co-cultures were treated with or without PM2.5 $(50 \mu \mathrm{g} / \mathrm{ml})$ for $4 \mathrm{~h}$.

\section{Preparation of $\mathrm{OA} \beta$}

oA $\beta$ was prepared as previously described [21, 22]. Briefly, oA $\beta 1-42$ was prepared by dissolving $A \beta 1-42$ to $1 \mathrm{mmol} / \mathrm{l}$ in $100 \%$ 1,1,1,3,3,3-hexafluoro-2-propanol. 1,1,1,3,3,3-Hexafluoro-2-propanol was dried by a vacuum desiccator and resuspended to $5 \mathrm{mmol} / \mathrm{l}$ in DMSO. To form oligomers, the amyloid peptide was diluted to a final concentration of $100 \mathrm{mmol} / \mathrm{l}$ with Ham's F-12, incubated at $4{ }^{\circ} \mathrm{C}$ for $24 \mathrm{~h}$ and then immediately added to cultures at a final concentration $5 \mu \mathrm{mol} / \mathrm{l}$. Formation of oA $\beta$ was confirmed by western blotting as previously described $[21,22]$.

\section{Flow cytometry for cell apoptosis, intracellular reactive oxygen species level, and mitochondrial ROS level}

To evaluate apoptosis, annexin V/propidium iodide (PI) analyses were performed as previously described [23]. Briefly, $5 \times 10^{5}$ cells were collected by centrifugation and resuspended in $500 \mu \mathrm{l}$ binding buffer. Then, $5 \mu \mathrm{l}$ annexin V and $10 \mu \mathrm{l}$ PI were added. After incubation at room temperature for $5 \mathrm{~min}$ in the dark, cells were analyzed by flow cytometry. Data were analyzed using CellQuest Pro software (BD).

The level of intracellular ROS was measured by DCFH oxidation using a commercial detection kit (Beyotime Biotechnology, Shanghai, China), as previously reported [24]. Briefly, $10 \mathrm{mM}(0.1 \mathrm{ml})$ DCFH-DA was diluted 1000 -fold in DMEM without serum, to give a $10 \mu \mathrm{M}$ concentration of DCFH-DA. The treated cells were washed three times with $\mathrm{PBS}$ and then incubated in $10 \mathrm{mM}$ DCFH-DA for $30 \mathrm{~min}$ at $37{ }^{\circ} \mathrm{C}$ without light. After being washed three times with PBS, cells were analyzed by flow cytometry. Data were analyzed using CellQuest Pro software (BD).

The level of mitochondrial ROS was measured by MitoSOX Red superoxide indicator staining (Invitrogen, Carlsbad, CA, USA), as previously reported [22]. Briefly, $50 \mu \mathrm{g}$ MitoSOX Red superoxide indicator was dissolved in $13 \mu \mathrm{l}$ DMSO to make $5 \mathrm{mM}$ MitoSOX reagent stock solution. Then, the stock solution was diluted 1000 -fold in buffer to make a $5 \mu \mathrm{M}$ MitoSOX reagent working solution. Microglia seeded $\left(5 \times 10^{5}\right)$ in 24-well plates were exposed to $1 \mathrm{ml}$ MitoSOX reagent working solution and then incubated for $10 \mathrm{~min}$ at $37{ }^{\circ} \mathrm{C}$. Cells were collected and washed twice by buffer, and cells were analyzed by flow cytometry. Data were analyzed using CellQuest Pro software (BD).

\section{MTT assay}

The MTT assay was performed using a Cell Counting Kit (Beyotime Biotechnology, Shanghai, China) as described previously [23]. Briefly, neurons were seeded at a density of $2 \times 10^{5}$ cells $/ \mathrm{cm}^{2}$ and received different treatments. Then, $10-\mu \mathrm{l}$ CCK- 8 solution was added to each well. Absorbance at $450 \mathrm{~nm}$ was measured $2 \mathrm{~h}$ after incubation in the dark.

\section{In vitro validation of oxidative capacity}

In vitro validation of oxidative capacity was performed as described previously [17]. Primary microglial cells were seeded at a density of $5 \times 10^{4}$ cells per dish. Cells were washed three times with PBS and incubated with a cell-permeable redox-sensitive fluorescent dye (CellROX, Invitrogen), followed by washes with PBS. Live-cell confocal imaging was performed within 30 min of washes. Signal intensity per unit area was determined for three fields of view from three different samples.

\section{Enzyme-linked immunosorbent assay}

Mouse IL-1 $\beta$ ELISA Kits were obtained from Invitrogen (Carlsbad, CA, USA). ELISA was performed according to manufacturer's instruction.

\section{Western blotting}

The following primary antibodies were used: mouse anti-Caspase-1 (1:200; Santa Cruz Biotechnology, Santa Cruz, CA, USA), rabbit anti-NLRP3 antibody (1:800; Santa Cruz Biotechnology, Santa Cruz, CA, USA), and mouse anti- $\beta$-actin antibody (1:1000; Santa Cruz Biotechnology, Santa Cruz, CA, USA).

Western blotting was performed as described elsewhere [25]. Cells were lysed in radio-immunoprecipitation assay 
(RIPA) lysis buffer supplemented with protease inhibitors (Complete; Roche, Indianapolis, IN, USA). The lysates were resolved by SDS-PAGE. Supernatants and the final pellets from each sample were heat-blocked for $5 \mathrm{~min}$ in loading buffer (125 mM Tris- $\mathrm{HCl}, 20 \%$ glycerol, 10\% 2-mercaptoethanol, 4\% SDS, $0.02 \%$ bromophenol blue, $\mathrm{pH}$ 6.8) and then subjected to electrophoresis on 10-20\% Tris-glycine SDS-PAGE gels. Proteins were then electrically transferred to a transfer membrane (BioRad, Hercules, CA, USA) and blocked for $1 \mathrm{~h}$ in Tris-HCl-buffered saline containing $5 \%$ skim milk and $0.1 \%$ Tween. The membranes were incubated in primary antibodies at $4{ }^{\circ} \mathrm{C}$ overnight in TBS buffer containing 5\% bovine albumin. The membranes were then rinsed with TBS buffer containing $0.1 \%$ Tween 20 , incubated with horseradish peroxidase (HRP)-labeled secondary antibody, and then stained with detection reagents. Finally, the membranes were developed using the enhanced chemiluminescence (ECL) system. Immunoreactivity was quantified using ImageJ software (Rasband, MD, USA).

\section{Measurement of caspase- 1 activity}

Microglia cells, seeded at a density of $1 \times 10^{7}$ cells and treated as described, were measured for caspase- 1 activity according to the manufacturer's instruction (Beyotime Biotechnology, Shanghai, China).

\section{Measurement of NADPH oxidase activity}

$\mathrm{NADPH}$ oxidase activity was measured by lucigeninenhanced chemiluminescence, as previously reported [26]. Microglial cells were collected, and total protein concentration was determined using a bicinchoninic acid protein assay kit (Beyotime Biotechnology, Shanghai, China). One hundred micromolars of $\mathrm{NADPH}$ and $5 \mu \mathrm{M}$ dark-adapted lucigenin were added into $0.5-\mathrm{ml}$ microcentrifuge tubes just before reading. Light emission was recorded over $5 \mathrm{~min}$, and values were expressed as mean light units per minute per milligram of protein. Using this method, the superoxide anion production also represents NADPH oxidase activity.

\section{Statistical analysis}

Statistical analysis was performed using SPSS software (version 17.0, SPSS, Chicago, IL, USA). All data are expressed as mean \pm SEM. Statistical significance was assessed by one-way ANOVA followed by the LSD procedure. If variances were unequal, the Games-Howell procedure was followed by ANOVA. The level of statistical significance was defined as $P<0.05$.

\section{Results}

PM2.5 exposure aggravates $\mathrm{A} A$-induced neuronal injury and inflammation in neurons-microglia co-cultures via increasing IL- $1 \beta$ production

First, we determined the effects of PM2.5 exposure on neuronal injury in neurons-microglia co-cultures by using transwell co-culture system. Neurons in the neuronal medium were plated in the lower chamber. Before co-culture, microglia were activated with LPS $(1 \mu \mathrm{g} / \mathrm{ml})$ for $3 \mathrm{~h}$ (LPS priming). Inserts containing microglia were washed with fresh serum-free DMEM and then plated in the upper chamber. Then, neurons-microglia co-cultures were treated with or without oA $\beta(5 \mu \mathrm{M})$. After $12 \mathrm{~h}$, neurons-microglia co-cultures were treated with or without PM2.5 $(50 \mu \mathrm{g} / \mathrm{ml})$ for $4 \mathrm{~h}$. We measured neuronal apoptosis using flow cytometry with annexin V/PI staining. When compared with neurons in co-cultures with LPS-priming microglia, oA $\beta$ stimulation and oA $\beta$-PM2.5 dual stimulation increased neuronal apoptosis $(\mathrm{oA} \beta$ stimulation: 18.86 vs. $6.77 \%, P<0.05$; oA $\beta-P M 2.5$ dual stimulation: 50.02 vs. $6.77 \%, P<0.05$; Fig. 1a, b). When compared with neurons in co-cultures with oA $\beta$ stimulated microglia, PM2.5 exposure increased neuronal apoptosis (50.02 vs. $18.86 \%, P<0.05$; Fig. 1a, b). To validate the association between PM2.5 and neuronal viability, we then detected neuronal viability via MTT assay. As expected, when compared with neurons in cocultures with LPS-priming microglia, oA $\beta$ stimulation and oA $\beta$-PM2.5 dual stimulation decreased neuronal viability by $19.42 \%(P<0.05)$ and $45.14 \%(P<0.05)$ (Fig. 1c). When compared with neurons in co-cultures with oA $\beta$-stimulated microglia, PM2.5 exposure decreased neuronal viability by $25.72 \%$ (Fig. 1c).

Then, we assessed whether PM2.5 induced inflammation in $\mathrm{oA} \beta$-stimulated microglia. Microglia were activated with LPS $(1 \mu \mathrm{g} / \mathrm{ml})$ for $3 \mathrm{~h}$ (LPS priming), and then $\mathrm{oA} \beta(5 \mu \mathrm{M})$ was added into the culture medium. After $12 \mathrm{~h}$, the cells were stimulated with PM2.5 $(50 \mu \mathrm{g} / \mathrm{ml})$ for $4 \mathrm{~h}$. The concentration of IL- $1 \beta$ in the culture supernatant was then measured by ELISA. As shown in Fig. 1d, PM2.5 exposure increased IL-1 $\beta$ concentration by $97.80 \%$ in oA $\beta$-stimulated microglia. Meanwhile, oA $\beta$ alone did not induce IL-1 $\beta$ production as previously described (Fig. 1d). Further, the effects of PM2.5 exposure on IL- $1 \beta$ production were dose-dependent (Fig. 1e). We also assessed whether IL-1 receptor antagonist (IL-1ra) affected the apoptosis or viability of the co-cultured neurons. In transwell co-culture system, PM2.5-oA $\beta$ stimulated neurons-microglia co-cultures were treated with or without IL-1ra. IL-1ra reduced neuronal apoptosis by $11.62 \%$ (Fig. 1a, b). Meanwhile, IL-1ra increased neuronal viability by $11.90 \%$ (Fig. 1c). Taken together, these results indicated that PM2.5 exposure aggravated oA $\beta$-induced neuronal injury and 


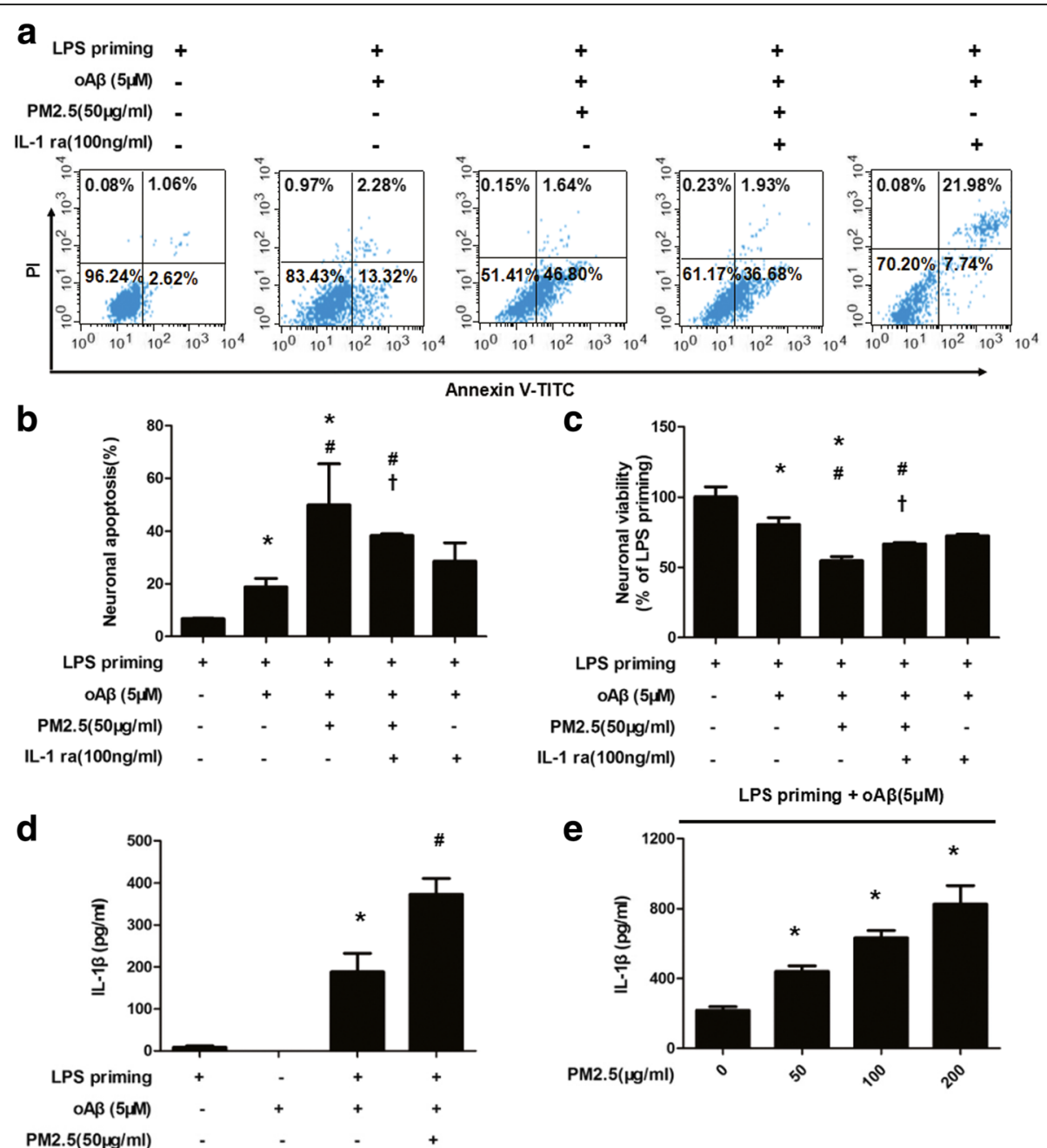

Fig. 1 PM2.5 exposure aggravates oAß-induced neuronal injury and inflammation in neurons-microglia co-cultures via increasing IL-1 3 production. In transwell co-culture system, neurons in the neuronal medium were plated in the lower chamber. Before co-culture, microglia were activated with LPS $(1 \mu \mathrm{g} / \mathrm{ml})$ for $3 \mathrm{~h}$ (LPS priming). Inserts containing microglia were washed with fresh serum-free DMEM and then plated in the upper chamber. Then, neurons-microglia co-cultures were treated with or without OAB $(5 \mu \mathrm{M})$. After $12 \mathrm{~h}$, neurons-microglia co-cultures were treated with or without PM2.5 $(50 \mathrm{\mu g} / \mathrm{ml})$ for $4 \mathrm{~h}$. a, b Apoptosis of co-cultured neurons was evaluated by flow cytometry with annexin V/PI staining. c Cell viability of co-cultured neurons was assessed via MTT assay. ${ }^{*} P<0.05$ vs. neurons in co-cultures with LPS-primed microglia. ${ }^{\#} P<0.05$ vs. neurons in co-cultures with oA $\beta$ stimulated microglia. ${ }^{\dagger} P<0.05$ vs. neurons in co-cultures with PM2.5-oA -stimulated microglia. d Microglia were activated with LPS $(1 \mu \mathrm{g} / \mathrm{ml})$ for $3 \mathrm{~h}$ (LPS priming), and then $\mathrm{AA} \beta(5 \mu \mathrm{M})$ was added into the culture medium. After $12 \mathrm{~h}$, the cells were stimulated with PM2.5 $(50 \mu \mathrm{g} / \mathrm{ml})$ for $4 \mathrm{~h}$. The concentration of IL-1 $\beta$ in the culture supernatant was then measured by ELISA. e The cells were stimulated with PM2.5 $(0,50,100,200 \mu \mathrm{g} / \mathrm{ml})$ for $4 \mathrm{~h}$. The concentration of IL-1 $\beta$ in the culture supernatant was measured by ELISA. All figures are representative of three independent experiments, performed in triplicate. ${ }^{*} P<0.05$ vs. LPS-primed microglia. ${ }^{\#} P<0.05$ vs. LPS-primed microglia stimulated with $\circ A \beta$

inflammation in neurons-microglia co-cultures via increasing IL-1 $\beta$ production.

\section{PM2.5-induced IL-1 $\beta$ production in oA $\beta$-stimulated} microglia is possibly dependent on NLRP3 inflammasome activation

We next examined whether NLRP3 inflammasome activation was involved in the PM2.5-induced IL-1 $\beta$ production. We first assessed whether LPS or oA $\beta$ alone induced IL-1 $\beta$ production in microglia. Microglia were stimulated by LPS or oA $\beta$ alone for varying time, and the concentration of IL-1 $1 \beta$ in the culture supernatant was measured by ELISA. We found that LPS stimulation alone for $0-12 \mathrm{~h}$ failed to induce IL-1 $\beta$ production. We detected IL-1 $\beta$ production when microglia were stimulated by LPS alone for $24 \mathrm{~h}$ (Fig. 2a). Meanwhile, we found that $\mathrm{OA} \beta$ stimulation alone for $0-24 \mathrm{~h}$ failed to induce IL-1 $\beta$ production (Fig. 2a). Then, LPS-primed microglia were stimulated with oA $\beta$ for $12 \mathrm{~h}$ and treated with pan-caspase inhibitor Z-VAD-FMK or caspase-1 inhibitor Z-YVAD-FMK for $30 \mathrm{~min}$ before PM2.5 exposure. After PM2.5 exposure for $4 \mathrm{~h}$, IL-1 $\beta$ concentration 
a

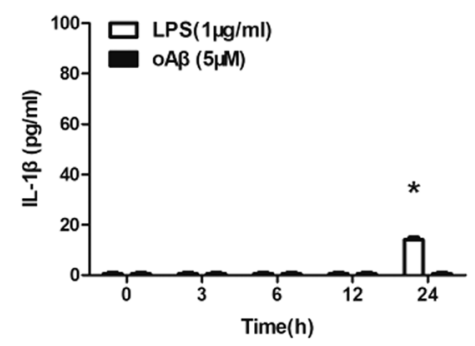

C

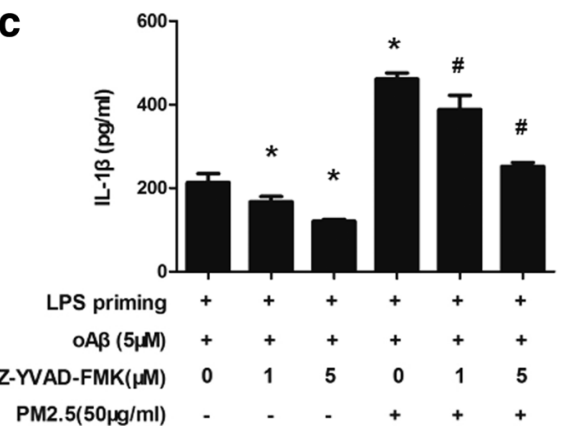

e

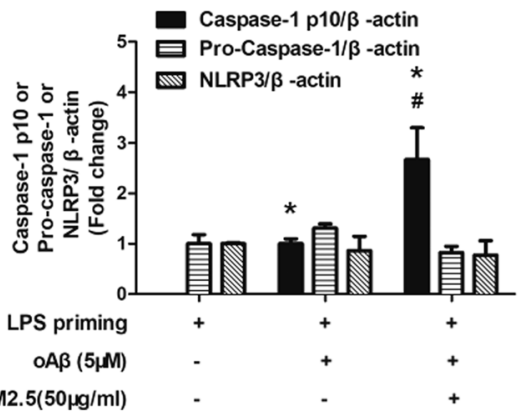

b

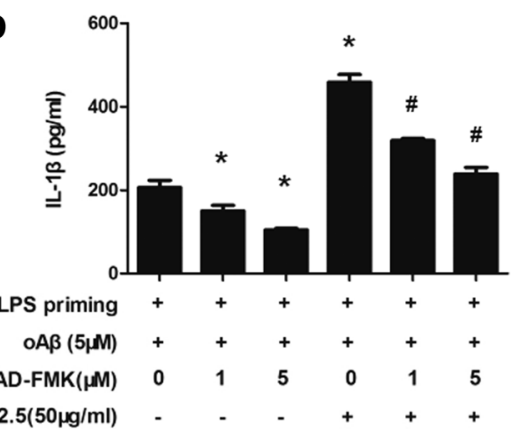

d
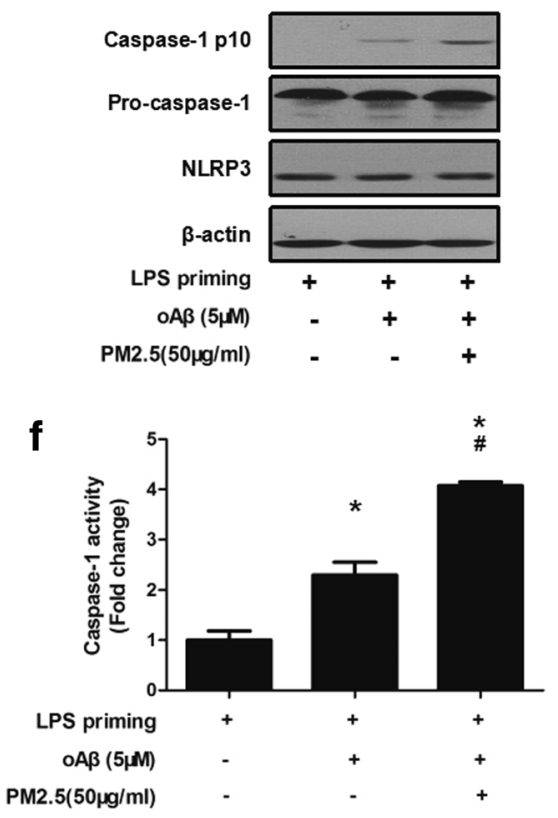

Fig. 2 PM2.5-induced IL-1 $\beta$ production in oA -stimulated microglia is possibly dependent on NLRP3 inflammasome activation. Microglia were stimulated by LPS or OA $\beta$ alone for varying time, and the concentration of IL-1 $\beta$ in the culture supernatant was measured by ELISA (a). Microglia primed with LPS for $3 \mathrm{~h}$ were washed with fresh serum-free DMEM. LPS-primed microglia were stimulated with OA 3 for $12 \mathrm{~h}$ and treated with pan-caspase inhibitor Z-VAD-FMK (b) or caspase-1 inhibitor Z-WAD-FMK (c) for 30 min before PM2.5 exposure. After PM2.5 exposure for $4 \mathrm{~h}$, IL-1 $\beta$ concentration in the culture supernatant was measured by ELISA. All figures are representative of three independent experiments, performed in triplicate. ${ }^{*} P<0.05$ vs. LPS-primed microglia stimulated with OA ${ }^{\#} P<0.05$ vs. LPS-primed microglia stimulated with $O A \beta$ and PM2.5. d, e LPS-primed microglia were stimulated with oA $\beta$ for $12 \mathrm{~h}$ and then treated with PM2.5 for $4 \mathrm{~h}$. The protein levels of Caspase-1 p10, pro-caspase-1, and NLRP3 were assessed by western blotting. $\beta$-actin was used as loading control. $\mathbf{f}$ LPS-primed microglia were stimulated with $O A \beta$ for $12 \mathrm{~h}$ and then treated with PM2.5 for $4 \mathrm{~h}$. Caspase- 1 activity was measured. All figures are representative of three independent experiments, performed in triplicate. ${ }^{*} P<0.05$ vs. LPS-primed microglia. ${ }^{\#} P<0.05$ vs. LPS-primed microglia stimulated with $O A \beta$

in the culture supernatant was measured. Both Z-VADFMK and Z-YVAD-FMK dose-dependently decreased IL-1 $\beta$ production in the culture supernatant (Fig. 2b, c). Next, we assessed the cleaved fraction of caspase-1 (Casp-1 p10), procaspase-1, and NLRP3 by western blotting. When compared with LPS-primed microglia, both oA $\beta$ stimulation and PM2. 5 -oA $\beta$ dual stimulation induced the cleavage of caspase- 1 and generated caspase-1 p10 subunit (Fig. 2d, e). When compared with the oA $\beta$-stimulated microglia, PM2.5 exposure increased the generation of caspase- 1 p10 subunit by nearly 2.76 -fold $(P<0.05)$ (Fig. $2 \mathrm{~d}$, e). We did not observe significant changes in pro-caspase-1 or NLRP3 protein expression after $\mathrm{oA} \beta$ stimulation or PM2.5-oA $\beta$ dual stimulation (Fig. 2d, e). To further the effects of PM2.5 exposure on NLRP3 inflammasome activation, we detected caspase-1 activity in microglia. When compared with the LPS-primed microglia, both $\mathrm{oA} \beta$ stimulation and PM2.5-oA $\beta$ dual stimulation increased caspase- 1 activity by nearly 2.30 -fold $(P<0.05)$ and 4.07 -fold $(P<0.05)$, respectively (Fig. 2f). When compared with the oA $\beta$-stimulated microglia, PM2.5 exposure increased caspase-1 activity by nearly 1.77 fold $(P<0.05)$ (Fig. 2f). Taken together, these results 
suggested that PM2.5-induced IL-1 $\beta$ production in oA $\beta$-stimulated microglia is possibly dependent on NLRP3 inflammasome activation.

\section{PM2.5 exposure increases ROS level in oA $\beta$-stimulated microglia}

PM2.5 is reported to trigger oxidative stress, which is regarded as a danger signal for NLRP3 inflammasome activation. By using a cell-permeable redox-sensitive fluorescent dye (CellROX), we demonstrated that PM2.5 exposure increased staining intensity/unit area in oA $\beta$-stimulated microglia $(P<0.05$, Figs. 3 and $4 a)$. To validate the association between PM2.5 and oxidative stress, the degree of oxidative stress (indicated by intracellular ROS level) was detected using a commercial detection kit. When compared with LPS-primed microglia, both oA $\beta$ stimulation and PM2.5-oA $\beta$ dual stimulation increased intracellular ROS level by nearly 1.57 -fold $(P<0.05)$ and 2.38 -fold $(P<0.05)$, respectively (Fig. 4b). When compared with oA $\beta$ stimulated microglia, PM2.5 exposure increased intracellular ROS level by nearly 1.52 -fold $(P<0.05)$ (Fig. 4b). In order to identify the source of ROS, we detected mitochondrial ROS level using MitoSOX Red superoxide indicator by flow cytometry. When compared with LPS-primed microglia, both oA $\beta$ stimulation and PM2.5-oA $\beta$ dual stimulation increased mitochondrial ROS level by nearly 1.79 -fold $(P<0.05)$ and 2.39 -fold, respectively
(Fig. 4c). When compared with oA $\beta$-stimulated microglia, PM2.5 exposure increased mitochondrial ROS level by nearly 1.34 -fold $(P<0.05)$ (Fig. 4c). Meanwhile, NADPH oxidase activity was tested by lucigenin-enhanced chemiluminescence. When compared with LPS-primed microglia, both oA $\beta$ stimulation and PM2.5-oA $\beta$ dual stimulation increased NADPH oxidase activity by nearly 1.50 -fold $(P<0.05)$ and 1.96-fold, respectively (Fig. 4d). When compared with oA $\beta$-stimulated microglia, PM2.5 exposure increased mitochondrial ROS level by nearly 1.31 -fold $(P<0.05) \quad$ (Fig. 4d). Taken together, these results suggested that PM2.5 exposure increased ROS level in oA $\beta$-stimulated microglia via both mitochondrialmediated manner and NADPH oxidase-mediated manner.

ROS is required for PM2.5-induced IL-1 $\beta$ production and NLRP3 inflammasome activation in oA $\beta$-stimulated microglia

Next, we investigated the role of ROS in IL- $1 \beta$ production and NLRP3 inflammasome activation in PM2. 5-oA $\beta$-stimulated microglia. LPS-primed microglia were stimulated with oA $\beta$ for $12 \mathrm{~h}$. Then, microglia were treated with ROS scavenger NAC, NADPH oxidase inhibitor DPI, and mitochondria-targeted antioxidant MitoQ for $30 \mathrm{~min}$ before PM2.5 exposure. After PM2.5 exposure for $4 \mathrm{~h}$, IL-1 $\beta$ concentration

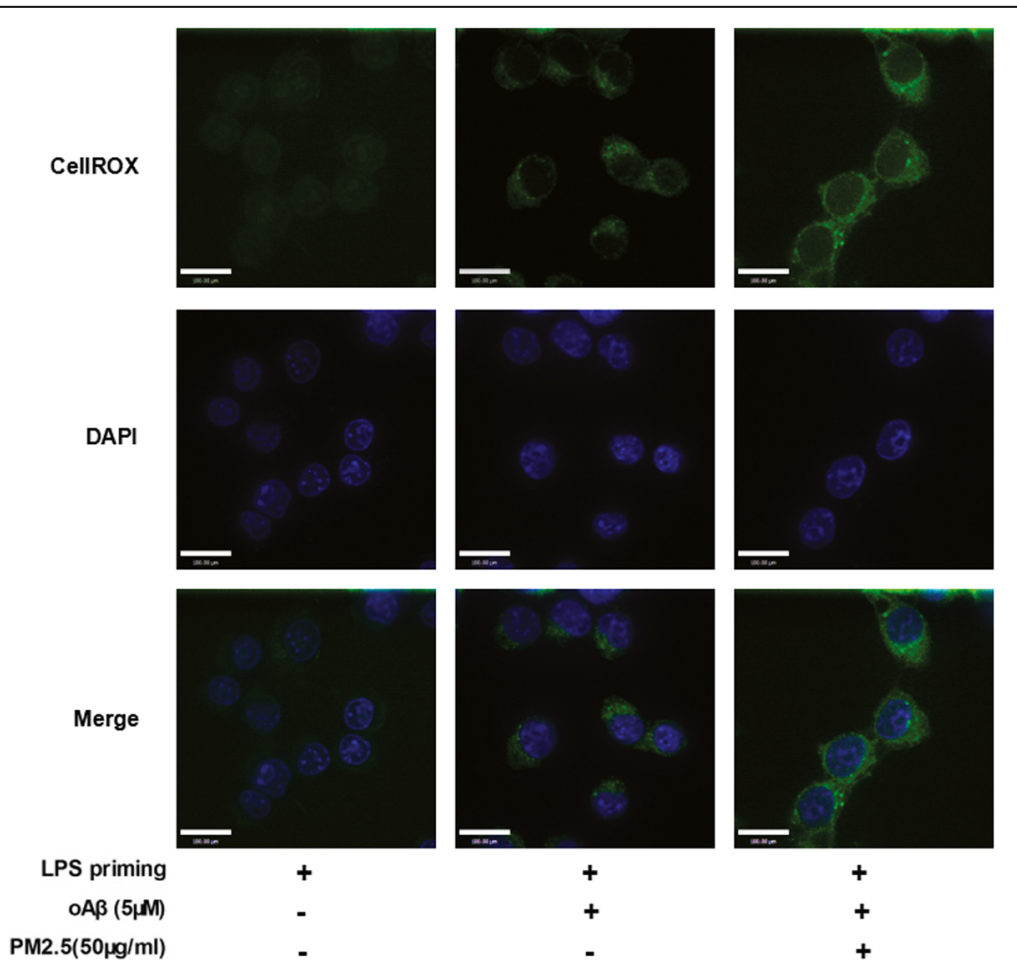

Fig. 3 PM2.5 exposure increases ROS level in oAß-stimulated microglia. Microglia with different treatment were incubated with CellROX (green). Meanwhile, cells were co-stained with DAPI (blue). Staining images of microglia with different treatments were shown. Scale bar $=20 \mu \mathrm{m}$ 

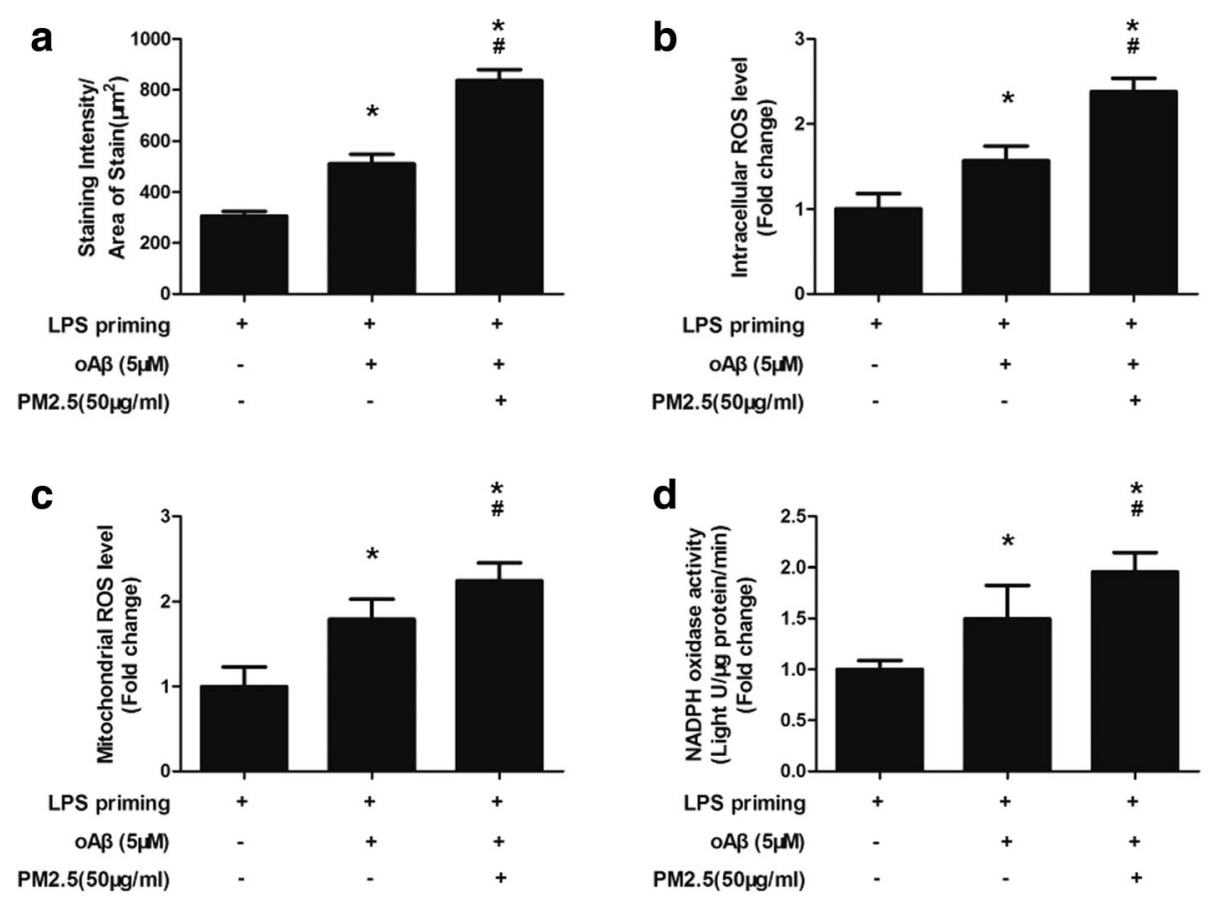

Fig. 4 PM2.5 exposure increases ROS level in oAß-stimulated microglia. Microglia with different treatment were incubated with CellROX. a Average staining intensity per unit area of stain quantified for three random fields of view. Microglia were treated with different stimulation, according to different groups. $\mathbf{b}$ The degree of oxidative stress (indicated by intracellular ROS level) was detected using a commercial detection kit. c The mitochondrial ROS level was detected by flow cytometry using MitoSOX Red superoxide indicator. $\mathbf{d}$ NADPH oxidase activity was tested by lucigenin-enhanced chemiluminescence. All figures are representative of three independent experiments, performed in triplicate. ${ }^{*} P<0.05$ vs. LPS-primed microglia. ${ }^{\#} P<0.05$ vs. LPS-primed microglia stimulated with $O A \beta$

and caspase- 1 activity in the culture supernatant were measured. NAC, DPI, and MitoQ reduced PM2.5-induced IL-1 $\beta$ production in oA $\beta$-stimulated microglia by $66.30 \%(P<0.05), 30.20 \%(P<0.05)$, and $52.59 \%$ $(P<0.05)$, respectively (Fig. 5a). Further, NAC, DPI, and MitoQ decreased PM2.5-induced caspase-1 activity in oA $\beta$-stimulated microglia by $58.59 \%(P<0$. $05)$, 31.42\% $(P<0.05)$, and $45.04 \%(P<0.05)$, respectively (Fig. $5 \mathrm{~b}$ ). Taken together, these results suggested that ROS was required for PM2.5-induced IL-1 $\beta$ production and NLRP3 inflammasome activation in oA $\beta$-stimulated microglia.

\section{$\mathbf{a}$}

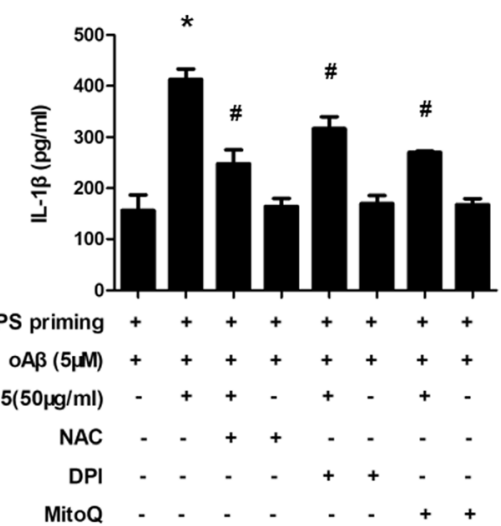

b

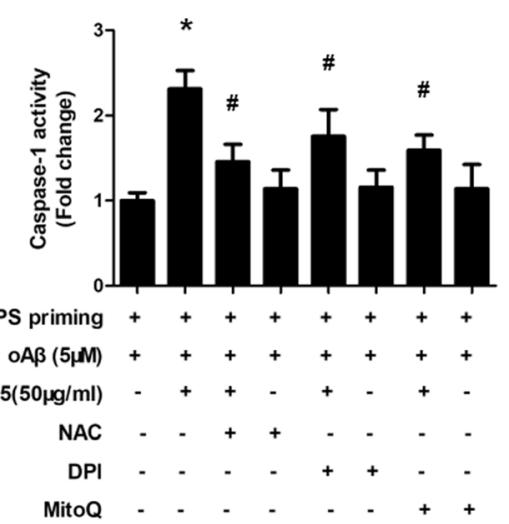

Fig. 5 ROS is required for PM2.5-induced IL-1 $\beta$ production and NLRP3 inflammasome activation in oA $\beta$-stimulated microglia. LPS-primed microglia were stimulated with oA $\beta$ for $12 \mathrm{~h}$. Then, microglia were treated with ROS scavenger NAC, NADPH oxidase inhibitor DPI, and mitochondria-targeted antioxidant MitoQ for 30 min before PM2.5 exposure for 4 h. a IL-1 3 concentration in the culture supernatant was measured by ELISA. b Caspase-1 activity was measured. All figures are representative of three independent experiments, performed in triplicate. * $P<0.05$ vs. LPS-primed microglia stimulated with oA $\beta .{ }^{\#} P<0.05$ vs. LPS-primed microglia stimulated with oA $\beta$ and PM2.5 
ROS and NLRP3 inflammasome activation is required for PM2.5-induced neuronal injury in neurons-microglia co-cultures

Further, we investigated the effects of ROS inhibitors and caspase- 1 inhibitors on neuronal injury in neuronsmicroglia co-cultures. In transwell co-culture system, neurons-microglia co-cultures were stimulated with oA $\beta$ for $12 \mathrm{~h}$. Then, the co-cultures were treated with ROS inhibitors and caspase- 1 inhibitors for $30 \mathrm{~min}$ before PM2.5 exposure. Z-VAD-FMK, Z-YVAD-FMK, NAC, DPI, and MitoQ were included. After PM2.5 exposure for $4 \mathrm{~h}$, apoptosis and viability of co-cultured neurons were detected. Z-VAD-FMK, Z-YVAD-FMK, NAC, DPI, and MitoQ reduced neuronal apoptosis by 12.48, 10.47, 12.10, 6.80, and 9.69\%, respectively (Figs. 6 and 7a). Meanwhile, Z-VAD-FMK, Z-YVAD-FMK, NAC, DPI, and MitoQ increased neuronal viability by $13.92,11.40$, $12.42,6.87$, and $11.55 \%$, respectively (Fig. 7b). Taken together, these results suggested that ROS and NLRP3 inflammasome activation was required for PM2.5-induced neuronal injury in neurons-microglia co-cultures.

\section{Discussion}

Firstly, we chose oA $\beta$-treated primary neurons-microglia co-cultures as an in vitro model of $\mathrm{AD}$ as previously reported [21, 22]. In the present study, we showed that PM2.5 exposure aggravated the oA $\beta$-induced neuronal injury in neurons-microglia co-cultures, as flow cytometry suggested that PM2.5 exposure increased neuronal apoptosis. A similar result was obtained by MTT assay, which revealed a corresponding decrease in neuronal viability following PM2.5 exposure. These results were

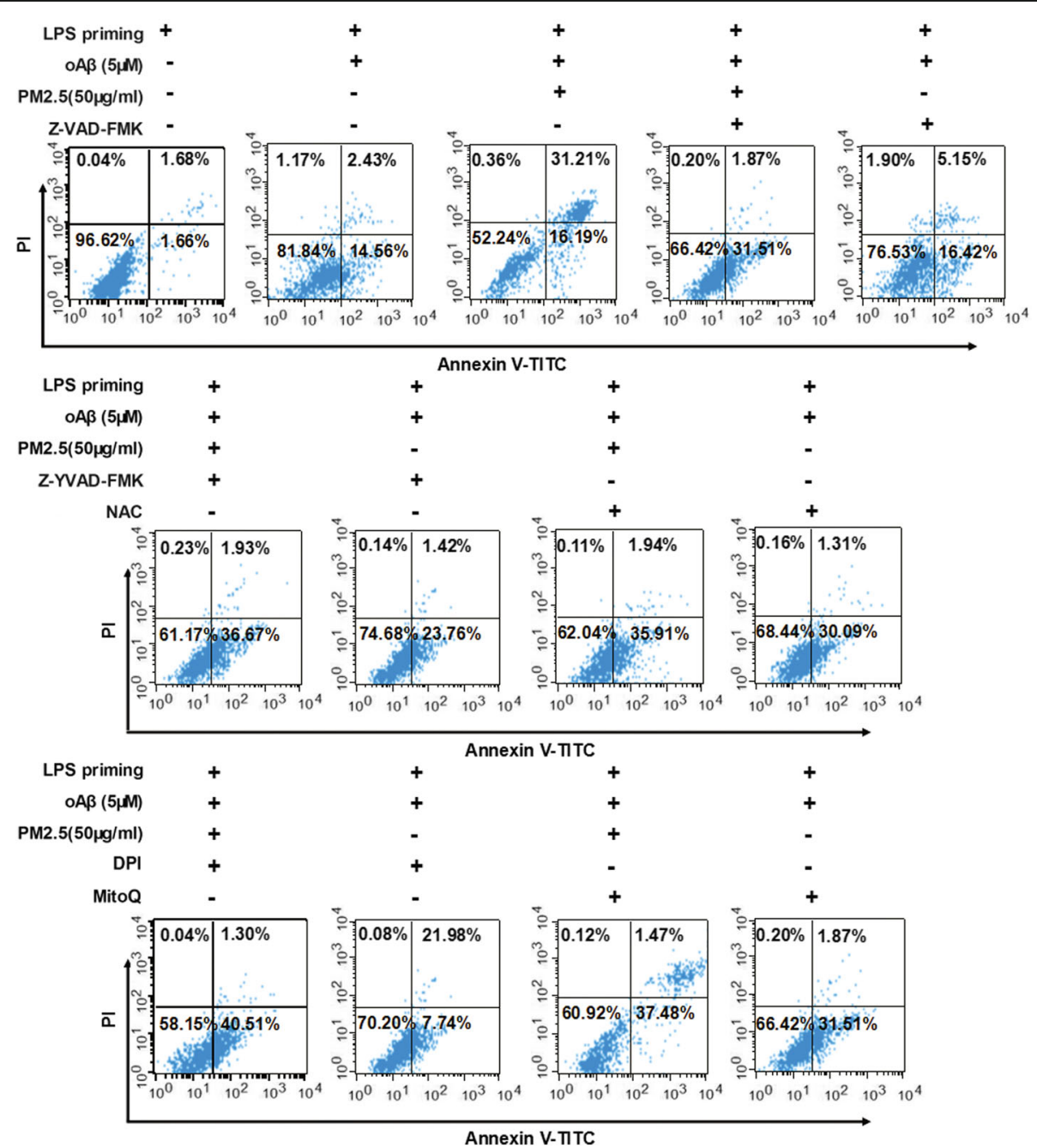

Fig. 6 ROS and NLRP3 inflammasome activation is required for PM2.5-induced neuronal injury in neurons-microglia co-cultures. In a transwell co-culture system, neurons-microglia co-cultures were stimulated with $\circ \mathrm{A} \beta$ for $12 \mathrm{~h}$ and treated with ROS inhibitors and caspase- 1 inhibitors for 30 min before PM2.5 exposure. Z-VAD-FMK, Z-YVAD-FMK, NAC, DPI, and MitoQ were included. After PM2.5 exposure for 4 h, cell apoptosis was assessed by annexin V/PI method, and a representative experiment is shown 
a

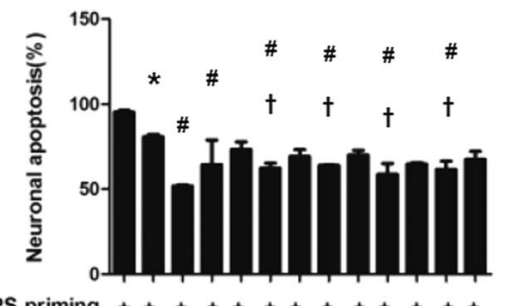

LPS priming +++++++++++++

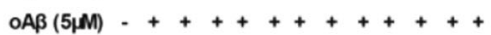

$\mathrm{PM} 2.5(50 \mu \mathrm{g} / \mathrm{ml}) \cdots++\cdot+\cdots+\cdots+\cdot+$

Z-VAD-FMK - - - + + - - - - .

Z-WAD-FMK - - - . + + . . .

NAC - - - - - + + - -

DPI - - - - - . + + -

MitoQ - . . . . . . + + b

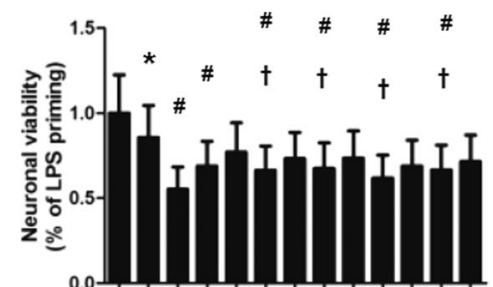

LPS priming +++++++++++++

$\mathrm{OA}(5, \mathrm{M})-+++++++++++$

$\mathrm{PM} 2.5(50 \mu \mathrm{g} / \mathrm{ml}) \cdots++\cdot+\cdots+\cdots+\cdot+$

Z-VAD-FMK - - - + + - - - - - -

Z-WVAD-FMK - - - . + + . . .

NAC - - - - . + + - -

DPI - - - - - - + + -

MitoQ - . . . . . . . + +

Fig. 7 ROS and NLRP3 inflammasome activation is required for PM2.5-induced neuronal injury in neurons-microglia co-cultures. In a transwell co-culture system, neurons-microglia co-cultures were stimulated with oA $\beta$ for $12 \mathrm{~h}$. Then, the co-cultures were treated with ROS inhibitors and caspase-1 inhibitors for 30 min before PM2.5 exposure for 4 h. Z-VAD-FMK, Z-YVAD-FMK, NAC, DPI, and MitoQ were included. a Apoptosis of co-cultured neurons were evaluated by flow cytometry with annexin V/PI staining. $\mathbf{b}$ Cell viability of co-cultured neurons were assessed via MTT assay. All figures are representative of three independent experiments, performed in triplicate. ${ }^{*} P<0.05$ vs. neurons in co-cultures with LPS-primed microglia. ${ }^{\#} P<0.05$ vs. neurons in co-cultures with oA $\beta$-stimulated microglia. ${ }^{\dagger} P<0.05$ vs. neurons in co-cultures with PM2.5-oA -stimulated microglia

in accordance with previous studies showing that PM2.5 led to neuronal injury $[27,28]$. Then, we showed that PM2.5 exposure increased IL-1 $\beta$ production in oA $\beta$ stimulated microglia. The result was supported by previous studies showing that PM2.5 caused IL- $1 \beta$ production in other cells, such as human airway epithelial cells, macrophage, ventricular myocytes, and a human keratinocyte cell line [17, 29-31]. More importantly, the effects of PM2.5 on the neuronal injury were mitigated by IL-1ra, as IL-1ra decreased neuronal apoptosis in flow cytometry and increased neuronal viability in MTT assay. The result was in agreement with a recent study from Dong et al. which showed that IL- $1 \beta$-induced inflammation triggered neuronal apoptosis [32]. To our knowledge, this is the first study showing that PM2.5 exposure aggravated neuronal injury and inflammation via increasing IL-1 $\beta$ production under AD context.

However, the underlying mechanisms by which PM2.5 increased IL- $1 \beta$ production in microglia are still not fully understood. IL-1 $\beta$ has been regarded as a biochemical marker of NLRP3 inflammasome activation [33]. In the subsequent experiment, we found that LPS stimulation alone for $0-12 \mathrm{~h}$ failed to induce IL-1 $\beta$ production. Meanwhile, we found that oA $\beta$ stimulation alone for $0-24 \mathrm{~h}$ failed to induce IL-1 $\beta$ production. This finding was in accordance with the previous studies [22]. In general, a two-signal model has been proposed for NLRP3 inflammasome activation. The first signal (priming) is provided by microbial or endogenous molecules that induce NLRP3 and pro-IL-1 $\beta$ expression through activation of NF- $\mathrm{kB}$; the second signal (activation) is triggered by ATP, pore-forming toxins, viral RNA, and particulate matter [34]. However, LPS is able to induce IL1 $\beta$ secretion and activation of inflammasome through non-canonical NLRP3 inflammasome activation and alternative NLRP3 inflammasome activation. In most studies, at least $6 \mathrm{~h}$ of LPS stimulation was needed to induce IL-1 $\beta$ secretion and activation of inflammasome in different cells [22, 35-37]. In the present study, $3 \mathrm{~h}$ of LPS stimulation was too short to induce IL-1 $\beta$ secretion and activation of inflammasome.

Then, we investigated whether NLRP3 inflammasome activation was involved in the PM2.5-induced IL-1 $\beta$ production. By using inhibitor, we found that both pancaspase inhibitor Z-VAD-FMK and caspase-1 inhibitor Z-YVAD-FMK dose-dependently decreased PM2.5-induced IL-1 $\beta$ production in oA $\beta$-stimulated microglia. More importantly, we observed a significant change in Casp-1 p10 protein expression in oA $\beta$-stimulated microglia after PM2.5 exposure. Meanwhile, PM2.5 exposure increased caspase- 1 activity in oA $\beta$-stimulated microglia. Based on the above evidence, we concluded that PM2.5induced IL-1 $\beta$ production in oA $\beta$-stimulated microglia was possibly dependent on NLRP3 inflammasome activation. This finding was in accordance with the previous studies conducted in human airway epithelial cells, macrophage, ventricular myocytes, and a human keratinocyte cell line [17, 29-31]. However, gene knockout experiments have not been performed in the present study. Without data of knockout experiments, no direct evidence shows that the effects of PM2.5 were mediated by NLRP3 inflammasome activation. On the other hand, further research is needed to investigate the role and mechanism of other cytokines in the effects of PM2.5. 
Increasing number of studies link oxidative stress to NLRP3 inflammasome activation in a wide range of autoinflammatory and autoimmune disorders [38]. Moreover, PM2.5 is demonstrated to trigger oxidative stress in some diseases, including cardiovascular diseases, respiratory diseases, diabetes mellitus, and Parkinson's disease [39-42]. As expected, in the present study, we showed that PM2.5 exposure increased ROS level in oA $\beta$-stimulated microglia, as intracellular ROS level was significantly increased following PM2.5 exposure. The enhancement in oxidative stress seemed to be mediated by the activation of NADPH oxidase and mitochondria damage. This finding was supported by previous reports showing that PM2.5 induced oxidative stress via both mitochondrial-mediated manner and NADPH oxidasemediated manner in human bronchial epithelial cells and human myeloid leukemia cells $[43,44]$.

Lastly, ROS inhibitors (NAC, DPI, and MitoQ) were used to investigate the role of ROS in IL- $1 \beta$ production and NLRP3 inflammasome activation. We found that all the ROS inhibitors decreased IL-1 $\beta$ production and caspase-1 activity in PM2.5-oA $\beta$-stimulated microglia. Meanwhile, we investigated the effects of ROS inhibitors and caspase- 1 inhibitors on neuronal injury in neuronsmicroglia co-cultures. We found that ROS inhibitors and caspase-1 inhibitors reduced PM2.5-induced neuronal injury. The results indicated that ROS played a key role in the PM2.5-induced IL-1 $\beta$ production and NLRP3 inflammasome activation. Furthermore, ROS and NLRP3 inflammasome activation was required for PM2.5-induced neuronal injury in neurons-microglia co-cultures.

\section{Conclusions}

Taken together, in this study, we have shown that PM2.5 exposure aggravated oA $\beta$-induced inflammation and neuronal injury in neurons-microglia co-cultures via increasing IL-1 $\beta$ production. Next, we demonstrated that PM2.5-induced IL-1 $\beta$ production in oA $\beta$-stimulated microglia was possibly dependent on NLRP3 inflammasome activation. Using ROS inhibitors, we demonstrated that ROS was required for PM2.5-induced IL-1 $\beta$ production and NLRP3 inflammasome activation in oA $\beta$ stimulated microglia. Lastly, we demonstrated that ROS and NLRP3 inflammasome activation was required for PM2.5-induced neuronal injury in neurons-microglia cocultures. These data provide in vitro evidence that PM2.5 exposure increased neuronal injury and inflammation in microglia under AD context. Importantly, we showed that inhibition of NLRP3 inflammasome prevented PM2.5-induced neuronal injury and inflammation, suggesting that it might be a useful therapeutic target in alleviating the harmful health effects of PM2.5 exposure. Meanwhile, the findings should be further confirmed in animal models of AD.

\section{Abbreviations}

AD: Alzheimer's disease; A 3 : Amyloid beta; IL-1 ra: IL-1 receptor antagonist; IL$1 \beta$ : Interleukin 1 beta; NLRP3: NLR family pyrin domain containing 3; oA : Oligomeric amyloid beta; PM2.5: Fine particulate matter; ROS: Reactive oxygen species

\section{Acknowledgements}

We gratefully acknowledge the funding support from National Natural Science Foundation of China (81500916, 81501092, 81271211, 81471215), Youth Medical Talents Program of "Science and Education Strong Health Project" of Jiangsu Province (QNRC2016079, QNRC2016068), Medical Innovation Team Program of "Science and Education Strong Health Project" of Jiangsu Province (CXTDA2017030), Natural Science Foundation of Jiangsu Province (BK20151592), and Social Development Project of Jiangsu Province (BE2015665, BE 2015715).

\section{Availability of data and materials}

The datasets used and/or analyzed during the current study are available from the corresponding author on reasonable request.

\section{Authors' contributions}

JQS, JX, YYT, and YDZ participated in designing the experiments. BRW, NNG, and ZO participated in carrying out the experiments. JQS, TJ, and JSZ participated in the data acquisition, analysis, and interpretation. BRW, JQS, JX and YYT participated in writing and revising the manuscript. All authors have read and approved the final manuscript.

\section{Ethics approval}

All animal procedures were approved by the Nanjing Medical University Experimental Animal Care and Use Committee.

\section{Competing interests}

The authors declare that they have no competing interests.

\section{Publisher's Note}

Springer Nature remains neutral with regard to jurisdictional claims in published maps and institutional affiliations.

\section{Author details}

${ }^{1}$ Department of Neurology, Jiangsu Geriatric Hospital, Nanjing 210024, Jiangsu Province, People's Republic of China. 'Department of Neurology, Nanjing First Hospital, Nanjing Medical University, No.68, Changle Road, Nanjing 210006, Jiangsu Province, People's Republic of China. ${ }^{3}$ Department of Neurology, Beijing Tiantan Hospital, Capital Medical University, No. 6 Tiantanxili, Dongcheng District, Beijing 100050, People's Republic of China. ${ }^{4}$ Jiangsu Key Laboratory of Integrated Traditional Chinese and Western Medicine for Prevention and Treatment of Senile Diseases, School of Medicine, Yangzhou University, Yangzhou 225001, Jiangsu Province, People's Republic of China. ${ }^{5}$ Department of Neurology, Northern Jiangsu People's Hospital, Clinical Medical College of Yangzhou University, Yangzhou 225001, Jiangsu Province, People's Republic of China.

Received: 16 January 2018 Accepted: 25 April 2018

Published online: 02 May 2018

\section{References}

1. Cummings JL, Cole G. Alzheimer disease. JAMA. 2002;287:2335-8.

2. Akimoto H. Global air quality and pollution. Science. 2003;302(5651):1716-9.

3. Yegambaram M, Manivannan B, Beach TG, Halden RU. Role of environmental contaminants in the etiology of Alzheimer's disease: a review. Curr Alzheimer Res. 2015;12:116-46.

4. Craig L, Brook JR, Chiotti Q, Croes B, Gower S, Hedley A, et al. Air pollution and public health: a guidance document for risk managers. J Toxicol Environ Health A. 2008;71:588-698.

5. Jung CR, Lin YT, Hwang BF. Ozone, particulate matter, and newly diagnosed Alzheimer's disease: a population-based cohort study in Taiwan. J Alzheimers Dis. 2015;44:573-84.

6. Spangenberg EE, Green KN. Inflammation in Alzheimer's disease: lessons learned from microglia-depletion models. Brain Behav Immun. 2017;61:1-11. 
7. Calderón-Garcidueñas L, Calderón-Garcidueñas A, Torres-Jardón R, AvilaRamírez J, Kulesza RJ, Angiulli AD. Air pollution and your brain: what do you need to know right now. Prim Health Care Res Dev. 2015;16:329-45.

8. Campbell A, Oldham M, Becaria A, Bondy SC, Meacher D, Sioutas C, et al. Particulate matter in polluted air may increase biomarkers of inflammation in mouse brain. Neurotoxicology. 2005;26:133-40.

9. Campbell A, Araujo JA, Li H, Sioutas C, Kleinman M. Particulate matter induced enhancement of inflammatory markers in the brains of apolipoprotein E knockout mice. J Nanosci Nanotechnol. 2009;9:5099-104.

10. Guerra R, Vera-Aguilar E, Uribe-Ramirez M, Gookin G, Camacho J, OsornioVargas AR, et al. Exposure to inhaled particulate matter activates early markers of oxidative stress, inflammation andunfolded protein response in rat striatum. Toxicol Lett. 2013;222:146-54

11. Ying Z, Xu X, Bai Y, Zhong J, Chen M, Liang $Y$, et al. Long-term exposure to concentrated ambient PM2.5 increases mouse blood pressure through abnormal activation of the sympathetic nervous system: a role for hypothalamic inflammation. Environ Health Perspect. 2014;122:79-86.

12. Bhatt DP, Puig KL, Gorr MW, Wold LE, Combs CK. A pilot study to assess effects of long-term inhalation of airborne particulate matter on early Alzheimer-like changes in the mouse brain. PLoS One. 2015;10:e0127102.

13. Shao BZ, Xu ZQ, Han BZ, Su DF, Liu C. NLRP3 inflammasome and its inhibitors: a review. Front Pharmacol. 2015;6:262.

14. Halle A, Hornung V, Petzold GC, Stewart CR, Monks BG, Reinheckel T, et al. The NALP3 inflammasome is involved in the innate immune response to amyloid-beta. Nat Immunol. 2008;9:857-65.

15. Heneka MT, Kummer MP, Stutz A, Delekate A, Schwartz S, Vieira-Saecker A, et al. NLRP3 is activated in Alzheimer's disease and contributes to pathology in APP/PS1 mice. Nature. 2013;493:674-8.

16. White CS, Lawrence CB, Brough D, Rivers-Auty J. Inflammasomes as therapeutic targets for Alzheimer's disease. Brain Pathol. 2017;27:223-2234.

17. Hirota JA, Hirota SA, Warner SM, Stefanowicz D, Shaheen F, Beck PL, et al. The airway epithelium nucleotide-binding domain and leucine-rich repeat protein 3 inflammasome is activated by urban particulate matter. J Allergy Clin Immunol. 2012;129:1116-25.

18. Hirota JA, Gold MJ, Hiebert PR, Parkinson LG, Wee T, Smith D, et al. The nucleotide-binding domain, leucine-rich repeat protein 3 inflammasome/IL1 receptor I axis mediates innate, but not adaptive, immune responses after exposure to particulate matter under $10 \mu \mathrm{m}$. Am J Respir Cell Mol Biol. 2015;52:96-105

19. Griciuc A, Serrano-Pozo A, Parrado AR, Lesinski AN, Asselin CN, Mullin K, et al. Alzheimer's disease risk gene CD33 inhibits microglial uptake of amyloid beta. Neuron. 2013;78:631-43.

20. Mizuno T, Kurotani T, Komatsu Y, Kawanokuchi J, Kato H, Mitsuma N, Suzumura A. Neuroprotective role of phosphodiesterase inhibitor ibudilast on neuronal cell death induced by activated microglia. Neuropharmacology. 2004:46:404-11.

21. Doi Y, Mizuno T, Maki Y, Jin S, Mizoguchi H, Ikeyama M, et al. Microglia activated with the toll-like receptor 9 ligand $C p G$ attenuate oligomeric amyloid \{beta\} neurotoxicity in in vitro and in vivo models of Alzheimer's disease. Am J Pathol. 2009;175:2121-32.

22. Parajuli $B$, Sonobe $Y$, Horiuchi $H$, Takeuchi $H$, Mizuno $T$, Suzumura A. Oligomeric amyloid $\beta$ induces $\mathrm{L}-1 \beta$ processing via production of ROS: implication in Alzheimer's disease. Cell Death Dis. 2013;4:e975.

23. Shi JQ, Chen J, Wang BR, Zhu YW, Xu Y, Wang J, et al. Short amyloid-beta immunogens show strong immunogenicity and avoid stimulating proinflammatory pathways in bone marrow-derived dendritic cells from C57BL/ 6J mice in vitro. Peptides. 2011;32:1617-25.

24. Block ML, Wu X, Pei Z, Li G, Wang T, Qin L, et al. Nanometer size diesel exhaust particles are selectively toxic to dopaminergic neurons: the role of microglia, phagocytosis, and NADPH oxidase. FASEB J. 2004;18(13):1618-20.

25. Shi JQ, Wang BR, Tian YY, Xu J, Gao L, Zhao SL, et al. Antiepileptics topiramate and levetiracetam alleviate behavioral deficits and reduce neuropathology in APPswe/PS1dE9 transgenic mice. CNS Neurosci Ther. 2013;19:871-81.

26. Liu D, Gao L, Roy SK, Cornish KG, Zucker IH. Role of oxidant stress on AT1 receptor expression in neurons of rabbits with heart failure and in cultured neurons. Circ Res. 2008;103:186-93.

27. Wei H, Feng Y, Liang F, Cheng W, Wu X, Zhou R, et al. Role of oxidative stress and DNA hydroxymethylation in the neurotoxicity of fine particulate matter. Toxicology. 2017;380:94-103.
28. Chen M, Li B, Sang N. Particulate matter (PM2.5) exposure seasondependently induces neuronal apoptosis and synaptic injuries. J Environ Sc (China). 2017;54:336-45.

29. Zhao Q, Chen $H$, Yang T, Rui W, Liu F, Zhang F, et al. Direct effects of airborne PM2.5 exposure on macrophage polarizations. Biochim Biophys Acta. 2016;1860:2835-43.

30. Grail A, Norval M. Significance of smoking and detection of serum antibodies to cytomegalovirus in cervical dysplasia. Br J Obstet Gynaecol. 1988;95:1103-10.

31. Zhang Y, Zheng L, Tuo J, Liu Q, Zhang X, Xu Z, et al. Analysis of PM2.5induced cytotoxicity in human $\mathrm{HaCaT}$ cells based on a microfluidic system. Toxicol in Vitro. 2017;43:1-8.

32. Dong Y, Kalueff AV, Song C. N-methyl-D-aspartate receptor-mediated calcium overload and endoplasmic reticulum stress are involved in interleukin-1 beta-induced neuronal apoptosis in rat hippocampus. J Neuroimmunol. 2017;307:7-13.

33. Song $L$, Pei $L$, Yao $S$, Wu Y, Shang $Y$. NLRP3 inflammasome in neurological diseases, from functions to therapies. Front Cell Neurosci. 2017;11:63.

34. He Y, Hara H, Núñez G. Mechanism and regulation of NLRP3 inflammasome activation. Trends Biochem Sci. 2016;41:1012-21.

35. Piccini A, Carta S, Tassi S, Lasiglié D, Fossati G, Rubartelli A. ATP is released by monocytes stimulated with pathogen-sensing receptor ligands and induces IL-1 beta and IL-18 secretion in an autocrine way. Proc Natl Acad Sci USA. 2008;105:8067-872.

36. Netea MG, Nold-Petry CA, Nold MF, Joosten LA, Opitz B, van der Meer JH, et al. Differential requirement for the activation of the inflammasome for processing and release of IL-1 beta in monocytes and macrophages. Blood. 2009;113:2324-35.

37. Kayagaki N, Warming S, Lamkanfi M, Vande Walle L, Louie S, Dong J, et al. Non-canonical inflammasome activation targets caspase-11. Nature. 2011; 479:117-21.

38. Abderrazak A, Syrovets T, Couchie D, El Hadri K, Friguet B, Simmet T, et al. NLRP3 inflammasome: from a danger signal sensor to a regulatory node of oxidative stress and inflammatory diseases. Redox Biol. 2015;4:296-307.

39. Feng S, Gao D, Liao F, Zhou F, Wang X. The health effects of ambient PM2.5 and potential mechanisms. Ecotoxicol Environ Saf. 2016;128:67-74.

40. Fiordelisi A, Piscitelli P, Trimarco B, Coscioni E, laccarino G, Sorriento D. The mechanisms of air pollution and particulate matter in cardiovascular diseases. Heart Fail Rev. 2017;22:337-47.

41. Kim HJ, Choi MG, Park MK, Seo YR. Predictive and prognostic biomarkers of respiratory diseases due to particulate matter exposure. J Cancer Prev. 2017; 22:6-15.

42. Lee PC, Raaschou-Nielsen O, Lill CM, Bertram L, Sinsheimer JS, Hansen J, et al. Gene-environment interactions linking air pollution and inflammation in Parkinson's disease. Environ Res. 2016;151:713-20.

43. Jin X, Su R, Li R, Song L, Chen M, Cheng L, et al. Amelioration of particulate matter-induced oxidative damage by vitamin $c$ and quercetin in human bronchial epithelial cells. Chemosphere. 2016;144:459-66.

44. Jin XT, Chen ML, Li RJ, An Q, Song L, Zhao Y, et al. Progression and inflammation of human myeloid leukemia induced by ambient PM2.5 exposure. Arch Toxicol. 2016;90:1929-38.

\section{Ready to submit your research? Choose BMC and benefit from:}

- fast, convenient online submission

- thorough peer review by experienced researchers in your field

- rapid publication on acceptance

- support for research data, including large and complex data types

- gold Open Access which fosters wider collaboration and increased citations

- maximum visibility for your research: over $100 \mathrm{M}$ website views per year

At BMC, research is always in progress.

Learn more biomedcentral.com/submissions 